\title{
Delineating Alzheimer's disease progression with MGAT3, a biomarker for improved prognosis and
} personalized therapy

\author{
"Clinical neurologists need a practical test to diagnose and prognosticate \\ Alzheimer's disease."
}

\section{KEYWORDS: Alzheimer's disease « bisdemethoxycurcumin $\approx$ MGAT3 biomarker - vitamin D3}

Studies of the neuronal mechanisms of Alzheimer's disease (AD) have discovered new therapeutic targets but not biomarkers for personalized medicine of AD. Exploitation of immune responses to amyloid- $\beta(A \beta)$ by peripheral blood mononuclear cells led to the discovery of two biomarkers: phagocytosis of amyloid- $\beta$ and transcription of MGAT3 (GnT-III). A pilot study suggests these two markers will be valuable for $\mathrm{AD}$ diagnosis, prognosis and therapy by vitamin D3 and curcuminoids [1].

\section{"The test of MGAT3 (GnT-III) transcription provides a more complex picture on the diagnosis, prognosis and therapy of $A D . "$}

Clinical neurologists need a practical test to diagnose and prognosticate AD. Early and significant loss of episodic memory is the core diagnostic criterion [2] but is difficult to apply without a longitudinal follow-up. The mini mental state examination is a rapid psychological test used in clinical practice that assesses memory, visualspatial orientation, registration, attention span and language skills. This test is helpful to demonstrate amnestic or nonamnestic presentation (the latter with language, visual-spatial or executive deficits) but is not specific for AD. Recently, the National Institute on Aging and The Alzheimer's Association workgroup proposed new criteria for AD dementia supported by 'AD biomarkers' [3]. These biomarkers are based on the tests of $A \beta$ and tau in the cerebrospinal fluid, PET amyloid imaging and quantitative MRI studies. However, in many patients, lumbar puncture and imaging are not an option and, furthermore, only verify the amyloidosis of the brain but not the antedating process. Therefore, an early blood biomarker would be advantageous to diagnose early stages of the disease in general practice.
Blood biomarkers have been based either on biochemical tests, such as plasma cytokine antibody assays, which require analysis of 18 signaling proteins [4], or functional tests, such as $A \beta$ phagocytosis and mRNA transcription by flow cytometry [5]. ApoE4 is a well-known risk factor for $\mathrm{AD}[6]$, and plasma apoE levels are decreased but not in all studies [7].

The multiple mechanisms common to neurodegenerative diseases, including inflammation, oxidative stress and dyslipidemia, render the goal of a specific protein blood biomarker elusive. In the brain, the defects in neuronal autophagy [8] and the retromer sorting pathway [9] are considered the crucial neuronal mechanisms leading to $\mathrm{AD}$. The mechanisms are postulated to include the disruption of substrate proteolysis within autolysosomes in the former and abnormal amyloid-precursor protein processing in the latter. However, these mechanisms cannot be examined in living patients by biochemical analysis, limiting their diagnostic value.

Here, we discuss an alternative approach based on a hypothesis that the immune system has a high sensitivity for the disease process, including abnormal degradation of $A \beta$ in lysosomes. Peripheral blood mononuclear cells (PBMCs) are defective in phagocytosis and degradation of $A \beta[5,10]$. In the AD brain, the distinction between macrophages and microglia is not clear but both appear to originate from the blood in pathological states (histochemistry shows macrophages migrating across a vessel wall [11] and mouse studies show the microglia to have bone-marrow origin [12]). The defects of AD macrophages also involve their trafficking - inability to emigrate across the blood-brain barrier and apoptotic death of macrophages engorged with $A \beta$ around brain vessels contributing to congophilic angiopathy [13]. According to this

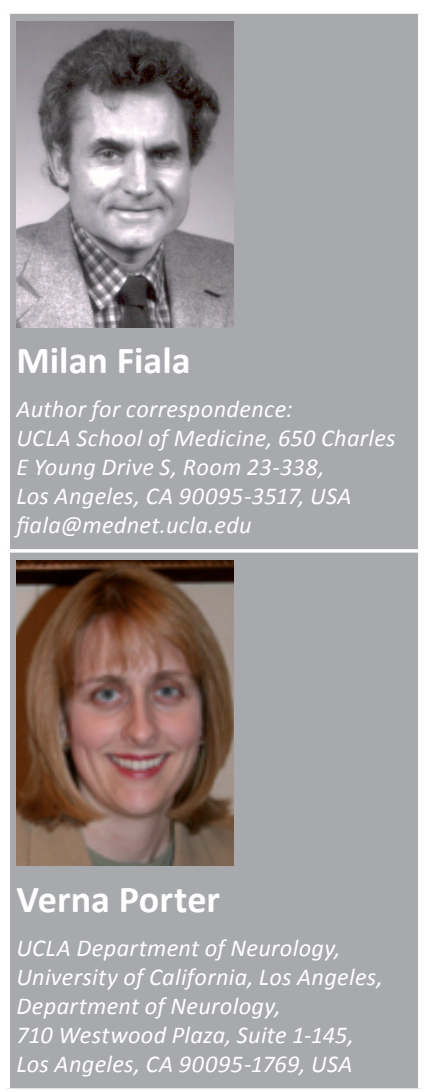

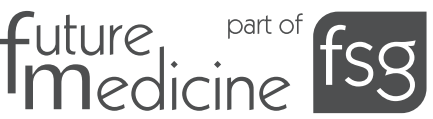


immune hypothesis, two functional biomarker tests have been developed by:

- Exposing freshly isolated PBMCs of AD patients to FAM-A $\beta$ ( $1 \mu \mathrm{g} / \mathrm{ml}$ overnight $)$ and testing the $A \beta$ uptake by flow cytometry;

- Challenging PBMCs with A $\beta(2 \mu \mathrm{g} / \mathrm{ml}$ overnight) and testing the transcription of $M G A T 3$ $(G n T-I I I)$ by reverse transcription PCR [5].

The flow cytometric test had a high sensitivity for AD (94\%) and less sensitivity for mild cognitive impairment (60\%), and a high specificity $(100 \%)$ in highly cognitively active subjects age-matched to AD patients, but extremely low specificity $(25 \%)$ in older caregivers. Therefore, a normal result of this test suggests the absence of $\mathrm{AD}$, whereas an abnormal result may indicate $\mathrm{AD}$ or retirement- and age-appropriate cognitive function with unknown prognosis for future dementia.

\section{"MGAT3 (GnT-III) transcription, by reverse transcription $P C R$, is a method for identifying three types of $A D$ patients possibly with a different prognosis and different therapeutic regimen..."}

The test of MGAT3 (GnT-III) transcription provides a more complex picture on the diagnosis, prognosis and therapy of $\mathrm{AD}$. In this test, PBMCs are challenged with $\mathrm{A} \beta \pm$ bisdemethoxycurcumin (BDC; a curcuminoid previously shown to improve $A \beta$ phagocytosis [10]). The results separate patients into type 0 (very low MGAT3 transcription, which is not improved by BDC), type I (low $M G A T 3$ transcription, which is upregulated by BDC) and type II (high MGAT3 transcription, which is downregulated by BDC). Although the rationale behind this test is not obvious, MGAT3 $(G n T-I I I)$ is an enzyme that plays a key role in the biosynthesis of $N$-glycans and the nervous system development [14]. Interestingly, the transcriptional regulation of Toll-like receptor-3 by $\mathrm{A} \beta$ and $\mathrm{BDC}$ demonstrates similar responses as MGAT3. Thus, the defect in MGAT3 may be associated with defects in other glycosylated proteins (such as Toll-like receptors) important for $A \beta$ phagocytosis.

In vitro the immune cell function was improved by BDC only in type I cells and by $1 \alpha, 25(\mathrm{OH})_{2}$ vitamin D3 in both type I and type II cells [15]. These results suggest that the diagnosis of type I and type II has significance for prognosis and therapy of AD. Our recent pilot study revealed that type 0 patients had worse 2-year prognosis for loss of independence than type I and type II patients $(p=0.009)$ [1]. A type II patient recovered from both cognitive dysfunction and defective $A \beta$ phagocytosis after surgery while taking vitamin D3 20,000 IU/week in addition to Aricept ${ }^{\circledR}$ (Eisai Co., Ltd, Tokyo, Japan), 10 mg orally every bedtime, Namenda ${ }^{\circledR}$ (Forest Laboratories, Inc., New York, NY, USA) 10 mg twice daily, aspirin $81 \mathrm{mg}$ orally daily, as well as Axona ${ }^{\circledR}$ (Accera, Inc., Broomfield, CO, USA) $40 \mathrm{~g}$ powder pack once daily. The patient's $A \beta$ phagocytosis was strongly (56-79\%) decreased by treatment of PBMCs with the specific inhibitor of $1 \alpha, 25(\mathrm{OH})_{2}$-vitamin D3 called ' $\mathrm{MK}$ ', indicating that her immune function was dependent upon $1 \alpha, 25(\mathrm{OH})_{2}$-vitamin D3.

\section{Conclusion}

MGAT3 (GnT-III) transcription, by reverse transcription PCR, is a method for identifying three types of $\mathrm{AD}$ patients possibly with a different prognosis and different therapeutic regimen, which must, however, be validated in future studies.

\section{Financial \& competing interests disclosure}

The authors have no relevant affiliations or financial involvement with any organization or entity with a financial interest in or financial conflict with the subject matter or materials discussed in the manuscript. This includes employment, consultancies, honoraria, stock ownership or options, expert testimony, grants or patents received or pending, or royalties.

No writing assistance was utilized in the production of this manuscript.

\section{Bibliography}

1 Fiala M, Mahanian M, Rosenthal M et al. MGAT3 mRNA: a biomarker for prognosis and therapy of Alzheimer's disease by vitamin $\mathrm{D}$ and curcuminoids. J. Alzheimers Dis. 25(1), 135-144 (2011).

2 Dubois B, Feldman HH, Jacova C et al. Research criteria for the diagnosis of Alzheimer's disease: revising the NINCDSADRDA criteria. Lancet Neurol. 6(8), 734-746 (2007).
3 McKhann GM, Knopman DS, Chertkow $\mathrm{H}$ et al. The diagnosis of dementia due to Alzheimer's disease: recommendations from the National Institute on Aging-Alzheimer's Association workgroups on diagnostic guidelines for Alzheimer's disease. Alzheimers Dement. 7(3), 263-269 (2011).

4 Ray S, Britschgi M, Herbert C et al. Classification and prediction of clinical Alzheimer's diagnosis based on plasma signaling proteins. Nat. Med. 13(11), 1359-1362 (2007).
5 Avagyan H, Goldenson B, Tse E et al. Immune blood biomarkers of Alzheimer disease patients. J. Neuroimmunol. 210(1-2), 67-72 (2009).

6 Saunders AM, Strittmatter WJ, Schmechel D et al. Association of apolipoprotein E allele $\varepsilon 4$ with late-onset familial and sporadic Alzheimer's disease. Neurology 43(8), 1467-1472 (1993).

7 Gupta VB, Laws SM, Villemagne VL et al. Plasma apolipoprotein E and Alzheimer disease risk: the AIBL study of aging. Neurology 76(12), 1091-1098 (2011). 
8 Nixon RA, Yang DS. Autophagy failure in Alzheimer's disease - locating the primary defect. Neurobiol. Dis. 43(1), 38-45 (2011).

9 Small SA, Kent K, Pierce A et al. Modelguided microarray implicates the retromer complex in Alzheimer's disease. Ann. Neurol. 58(6), 909-919 (2005).

10 Fiala M, Liu PT, Espinosa-Jeffrey A et al. Innate immunity and transcription of MGAT3 and Toll-like receptors in Alzheimer's disease patients are improved by bisdemethoxycurcumin. Proc. Natl Acad. Sci. USA 104(31), 12849-12854 (2007).
11 Fiala M, Liu QN, Sayre J et al. Cyclooxygenase-2-positive macrophages infiltrate the Alzheimer's disease brain and damage the blood-brain barrier. Eur. J. Clin. Invest. 32(5), 360-371 (2002).

12 Simard AR, Soulet D, Gowing G, Julien JP, Rivest $S$. Bone marrow-derived microglia play a critical role in restricting senile plaque formation in Alzheimer's disease. Neuron 49(4), 489-502 (2006).

13 Zaghi J, Goldenson B, Inayathullah M et al. Alzheimer disease macrophages shuttle amyloid- $\beta$ from neurons to vessels, contributing to amyloid angiopathy. Acta Neuropathol. 117(2), 111-124 (2009).

14 Shigeta M, Shibukawa Y, Ihara $\mathrm{H}$, Miyoshi E, Taniguchi N, Gu J. $\beta 1,4-N$ acetylglucosaminyltransferase III potentiates $\beta 1$ integrin-mediated neuritogenesis induced by serum deprivation in Neuro2a cells. Glycobiology 16(6), 564-571 (2006).

15 Masoumi A, Goldenson B, Ghirmai S et al. $1 \alpha, 25$-dihydroxyvitamin D3 interacts with curcuminoids to stimulate amyloid- $\beta$ clearance by macrophages of Alzheimer's disease patients. J. Alzheimers Dis. 17, 703-717 (2009). 